\title{
MINIJETS AND THE TWO-BODY PARTON CORRELATION
}

\author{
G. Calucci and D. Treleani \\ Dipartimento di Fisica Teorica dell'Università and INFN. \\ Trieste, I 34014 Italy
}

\begin{abstract}
A large number of double parton scatterings have been recently measured by CDF. The double parton scattering process measures $\sigma_{e f f}$, a non perturbative quantity related to the hadronic transverse size and with the dimensions of a cross section. The actual value measured by CDF is considerably smaller as compared with the naive expectation, namely the value of the inelastic non diffractive cross section. The small value of $\sigma_{\text {eff }}$ may be an effect of the hadron structure in transverse plane. We discuss the problem by taking into account, at all orders, the two-body parton correlations in the many-body parton distributions.
\end{abstract}




\section{Introduction}

CDF has recently shown evidence of a large number of double parton collisions[1], namely events where, in the same inelastic interaction, two different pairs of partons scatter independently with large momentum transfer. The inclusive cross section for a double parton scattering is written as[2]:

$$
\sigma_{D}=\int_{p_{t}^{c}} D_{2}\left(x_{A}, x_{A}^{\prime} ; \mathbf{b}\right) \hat{\sigma}\left(x_{A}, x_{B}\right) \hat{\sigma}\left(x_{A}^{\prime}, x_{B}^{\prime}\right) D_{2}\left(x_{B}, x_{B}^{\prime} ; \mathbf{b}\right) d \mathbf{b} d x_{A} d x_{B} d x_{A}^{\prime} d x_{B}^{\prime}
$$

$\hat{\sigma}\left(x_{A}, x_{B}\right)$ is the parton-parton cross section integrated with the cut off $p_{t}^{c}$, which is the lower threshold to observe final state partons as minijets, $x$ is the momentum fraction, $A$ and $B$ are labels to identify the two interacting hadrons. $\sigma_{D}$ is a function of the product $\hat{\sigma}\left(x_{A}, x_{B}\right) \hat{\sigma}\left(x_{A}^{\prime}, x_{B}^{\prime}\right)$. Actually the two different partonic interactions are localized in two regions in transverse space with a size of order $\left(1 / p_{t}^{c}\right)^{2}$ and at a relative distance of the order of the hadronic radius $R$, in such a way that the two partonic interactions add incoherently in the double scattering cross section. The non perturbative input in Eq.(1.1) is the two-body parton distribution $D_{2}\left(x, x^{\prime} ; \mathbf{b}\right)$, which depends on the fractional momenta of the two partons taking part to the interaction and on their relative transverse distance $\mathbf{b}$. The transverse distance $\mathbf{b}$ has to be the same for the two partons of hadron $A$ and the two partons of hadron $B$, in order to have the alinement which is needed for a double collision to occur. $D_{2}$ is a dimensional quantity and therefore the process introduces a non perturbative scale factor which is related to the hadronic transverse size.

The simplest possibility to consider is the one where the dependence of $D_{2}$ on the different variables is factorized:

$$
D_{2}\left(x, x^{\prime} ; \mathbf{b}\right)=f_{\text {eff }}(x) f_{\text {eff }}\left(x^{\prime}\right) F(\mathbf{b})
$$

$f_{\text {eff }}$ is the effective parton distribution, namely the gluon plus $4 / 9$ of the quark and anti-quark distributions and $F(\mathbf{b})$ is normalized to one. Multiparton distribution are then uncorrelated and $D_{2}$ does not contain further informations with respect to the one-body parton distribution (actually $f_{\text {eff }}$ ) apart form the dependence on $\mathbf{b}$, whose origin is the dimensionality of $D_{2}$ and which gives rise to the scale factor $\sigma_{e f f}$. In fact in this case one may write

$$
\sigma_{D}=\frac{\sigma_{S}^{2}}{\sigma_{e f f}}
$$

with

$$
\frac{1}{\sigma_{e f f}}=\int F^{2}(b) d^{2} b
$$


and

$$
\sigma_{S}=\int_{p_{t}^{c}} f_{e f f}\left(x_{A}\right) f_{e f f}\left(x_{B}\right) \hat{\sigma}\left(x_{A}, x_{B}\right) d x_{A} d x_{B},
$$

the single scattering expression of the perturbative QCD parton model.

Eq.(1.2) is the basic hypothesis underlying the signature of a double parton collision which one has been looking for in the experimental search $[1,3]$. The expected characteristic feature of a double collision is in fact that it should produce a final state analogous to the one obtained by super-posing two single scattering processes. CDF measures:

$$
\sigma_{e f f}=14.5 \pm 1.7_{-2.3}^{+1.7} m b
$$

By looking at the dependence of $\sigma_{\text {eff }}$ on $x$ CDF has been able to verify the correctness of the factorization hypothesis in Eq.(1.2). The range of values of $x$ available is limited to $x \leq .2$, for the interaction producing a pair of minijets, and to $x \leq .4$ for the interaction giving rise to a minijet and a photon. In the limited range of values of $x$ available, the factorization hypothesis has shown to be consistent with the experimental evidence.

Since the uncorrelation hypothesis, as expressed in Eq.(1.2), is not inconsistent with experiment, one can work out the case where all multiparton distributions are uncorrelated and one may look for the sum of all multiparton interactions to the hadronic inelastic cross section. The subset where all multiple parton collisions are disconnected can be easily summed up in the uncorrelated case[4]. The result is the semi-hard hadronic cross section $\sigma_{H}$, which represents the contribution to the hadronic inelastic cross section from events with at least one semi-hard partonic interaction. The actual expression is

$$
\sigma_{H}=\int d^{2} \beta\left[1-e^{-\sigma_{S} F(\beta)}\right]=\sum_{n=1}^{\infty} \int d^{2} \beta \frac{\left(\sigma_{S} F(\beta)\right)^{n}}{n !} e^{-\sigma_{S} F(\beta)}
$$

The integration on the impact parameter of the hadronic collision, $\beta$, gives the dimensionality to the cross section. The argument of the integral has the meaning of a Poissonian distribution of multiple semi-hard partonic interactions with average number depending on the impact parameter.

$\sigma_{e f f}$ is related to $\sigma_{H}$ through Eq.(1.6). Indeed the precise relation requires the knowledge of the functional dependence of $F(\beta)$. Still the expression in Eq.(1.6) shows that $\sigma_{H}$ does not depend on many details. The only relevant quantity is the value of $\beta$ which makes $F(\beta)$ small, in such a way that the overall argument of the integral becomes close to zero. In the simplest case where $F(\beta)=\exp \left(-\beta^{2} / R^{2}\right) / \pi R^{2}$ one obtains a closed analytic expression for $\sigma_{H}$. Actually

$$
\sigma_{H}=2 \pi R^{2}\left[\gamma+\ln \kappa+E_{1}(\kappa)\right]
$$


where $\gamma=0.5772 \ldots$ is Euler's constant, $\kappa=\sigma_{S} /\left(2 \pi R^{2}\right)$ and $E_{1}(x)$ is the exponential integral. For small $\kappa$ one obtains $\sigma_{H} \rightarrow 2 \pi R^{2} \kappa=\sigma_{S}$, for large $\kappa$, namely $\sigma_{S} \rightarrow \infty$, one obtains $\sigma_{H} \rightarrow 2 \pi R^{2}(\gamma+\ln \kappa)$. In this simplest example $\sigma_{e f f}=2 \pi R^{2}$. The value of $\sigma_{H}$ is therefore proportional to the measured value of $\sigma_{e f f}$, the proportionality factor is slightly dependent on energy and on the cutoff. Sensible values of the hadron-hadron c.m. energy and of the cutoff give values for $\sigma_{H}$ which are some $30-40 \%$ larger with respect to the value of $\sigma_{e f f}$. Although $\sigma_{H}$ has no reason in principle to be close to $\sigma_{\text {inel }}$, a value of $\sigma_{H}$ as small as $\sigma_{i n e l} / 2$, which would result from such considerations, seems nevertheless too small. Indeed $\sigma_{H}$, as given in Eq.(1.6), has a smooth dependence on the infrared cutoff. If one could really have the interaction under control also at small values of $p_{t}^{c}$ one would say that $\sigma_{H}$ has to be the same as the inelastic non diffractive cross section $\sigma_{i n e l}$. It would therefore be rather natural to expect for $\sigma_{H}$ a value not much smaller as compared to the value of $\sigma_{\text {inel }}$. The large difference between $\sigma_{H}$ and $\sigma_{\text {inel }}$ could therefore be an indication that the simplest assumptions underlying the derivation of the expression in Eq.(1.6) have to be revised.

The main hypothesis which has been done to obtain the expression for $\sigma_{H}$ in Eq.(1.6) is the Poissonian multiparton distribution. On the other hand one has to expect correlations between partons as a consequence of the binding force. While most probably correlations will affect the $x$ dependence of the multiparton distribution only for finite values of $x$, and therefore at large rapidities, correlations in the transverse parton coordinates are present in every kinematical regime. Indeed the main reason of interest in multiple parton collisions, besides the identification of the process itself, is precisely the measure of the many-body parton correlations, which is an information on the hadron structure independent on the one-body parton distributions usually considered in hard processes.

In the present paper we work out the most general expression for the semihard cross section $\sigma_{H}$, which one obtains by assuming that only two-body parton correlations are present in the many-body parton distributions and by summing all disconnected multiple parton interactions. The two body parton correlation in transverse plane is then worked out in great detail in a few cases by considering different explicit shapes.

\section{General formalism and semi-hard cross section}

At a given resolution, provided by the cut off $p_{t}^{\text {min }}$ that defines the lower threshold for the production of minijets, one can find the hadron in various partonic configurations. The probability of an exclusive $n$-parton distribution, namely the probability to find the hadron in a configuration with $n$-partons, is denoted by $W_{n}\left(u_{1} \ldots u_{n}\right)$. $u_{i} \equiv\left(\mathbf{b}_{i}, x_{i}\right)$ represents the transverse partonic coordinate $\mathbf{b}_{i}$ and longitudinal fractional momentum $x_{i}$ while color and flavour variables are not considered explicitly. The distributions are symmetric in the variables $u_{i}$. One defines the generating functional 
of the multiparton distributions as:

$$
\mathcal{Z}[J]=\sum_{n} \frac{1}{n !} \int J\left(u_{1}\right) \ldots J\left(u_{n}\right) W_{n}\left(u_{1} \ldots u_{n}\right) d u_{1} \ldots d u_{n}
$$

where the dependence on the infrared cutoff $p_{t}^{\text {min }}$ is implicitly understood, and one may introduce also the logarithm of the generating functional: $\mathcal{F}[J]=\ln (\mathcal{Z}[J])$. The conservation of the probability yields the overall normalization condition

$$
\mathcal{Z}[1]=1
$$

One may use the generating functional to derive the many body densities, i.e. the inclusive distributions $D_{n}\left(u_{1} \ldots u_{n}\right)$ :

$$
\begin{aligned}
D_{1}(u) & =W_{1}(u)+\int W_{2}\left(u, u^{\prime}\right) d u^{\prime}+\frac{1}{2} \int W_{3}\left(u, u^{\prime}, u^{\prime \prime}\right) d u^{\prime} d u^{\prime \prime}+\ldots \\
& =\left.\frac{\delta \mathcal{Z}}{\delta J(u)}\right|_{J=1}=\left.\frac{\delta \mathcal{F}}{\delta J(u)}\right|_{J=1}, \\
D_{2}\left(u_{1}, u_{2}\right) & =W_{2}\left(u_{1}, u_{2}\right)+\int W_{3}\left(u_{1}, u_{2}, u^{\prime}\right) d u^{\prime}+\frac{1}{2} \int W_{4}\left(u_{1}, u_{2}, u^{\prime}, u^{\prime \prime}\right) d u^{\prime} d u^{\prime \prime} \ldots \\
& =\left.\frac{\delta^{2} \mathcal{Z}}{\delta J\left(u_{1}\right) \delta J\left(u_{2}\right)}\right|_{J=1}=\left.\frac{\delta^{2} \mathcal{F}}{\delta J\left(u_{1}\right) \delta J\left(u_{2}\right)}\right|_{J=1}+\left.\frac{\delta \mathcal{F}}{\delta J\left(u_{1}\right)} \frac{\delta \mathcal{F}}{\delta J\left(u_{2}\right)}\right|_{J=1} \\
& \ldots
\end{aligned}
$$

The many body parton correlations are defined by expanding $\mathcal{F}[J]$ in the vicinity of $J=1$ :

$$
\begin{array}{r}
\mathcal{F}[J]=\int D(u)[J(u)-1] d u+\sum_{n=2}^{\infty} \frac{1}{n !} \int C_{n}\left(u_{1} \ldots u_{n}\right)\left[J\left(u_{1}\right)-1\right] \ldots \\
\ldots\left[J\left(u_{n}\right)-1\right] d u_{1} \ldots d u_{n}
\end{array}
$$

Here $D=D_{1}$ and the correlations $C_{n}$ describe how much the distribution deviates from a Poisson distribution, which corresponds in fact to $C_{n} \equiv 0, n \geq 2$.

By assuming the validity of the AGK cutting rules[5] for semi-hard interactions one may express the semi-hard cross section as the sum of all probabilities of multiple parton collisions:

$$
\sigma_{H}=\int d^{2} \beta \sigma_{H}(\beta)
$$

with 


$$
\begin{aligned}
\sigma_{H}(\beta)= & \int \sum_{n} \frac{1}{n !} \frac{\delta}{\delta J\left(u_{1}\right)} \cdots \frac{\delta}{\delta J\left(u_{n}\right)} \mathcal{Z}_{A}[J] \\
& \times \sum_{m} \frac{1}{m !} \frac{\delta}{\delta J^{\prime}\left(u_{1}^{\prime}-\beta\right)} \cdots \frac{\delta}{\delta J^{\prime}\left(u_{m}^{\prime}-\beta\right)} \mathcal{Z}_{B}\left[J^{\prime}\right] \\
& \times\left.\left\{1-\prod_{i=1}^{n} \prod_{j=1}^{m}\left[1-\hat{\sigma}_{i, j}\left(u, u^{\prime}\right)\right]\right\} \prod d u d u^{\prime}\right|_{J=J^{\prime}=0}
\end{aligned}
$$

where $\beta$ is the impact parameter between the two interacting hadrons $A$ and $B$ and $\hat{\sigma}_{i, j}$ is the elementary probability for parton $i$ (of $A$ ) to have a hard interaction with parton $j$ (of $B$ ). The semi-hard cross section is constructed summing over all possible partonic configurations of the two interacting hadrons (the sums over $n$ and $m$ ) and, for each configuration with $n$ partons from $A$ and $m$ partons from $B$, summing over all possible multiple partonic interactions. This last sum is constructed asking for the probability of no interaction between the two configurations ( actually $\prod_{i=1}^{n} \prod_{j=1}^{m}\left[1-\hat{\sigma}_{i, j}\right]$ ). One minus the probability of no interaction is equal to the sum over all semi-hard interaction probabilities.

The presence of multiple parton interactions is induced by the large flux of partons which is effective at large energies. The most important contribution to the semihard cross section, as a consequence, is the contribution of the disconnected partonic collisions, namely the interactions where each parton undergoes at most one semihard collision. These are, in fact, those multiple partonic interactions that, at a given number of partonic collisions, maximize the parton flux. We simplify therefore the problem by expanding the interaction probability ( the factor in curly brackets ) as sums and by removing all the addenda containing repeated indices:

$$
\left\{1-\prod_{i, j}^{n, m}\left[1-\hat{\sigma}_{i j}\right]\right\} \Rightarrow \sum_{i j} \hat{\sigma}_{i j}-\frac{1}{2 !} \sum_{i j} \sum_{k \neq i, l \neq j} \hat{\sigma}_{i j} \hat{\sigma}_{k l}+\ldots
$$

as a result the semi-hard cross section is constructed with multiple disconnected parton collisions only, where disconnected refers to the perturbative component of the interaction. Some features of semi-hard parton rescatterings are presented in Appendix A. Because of the symmetry of the derivative operators in Eq.(2.5) one can replace the expression in Eq.(2.6) with:

$$
n m \hat{\sigma}_{11}-\frac{1}{2 !} n(n-1) m(m-1) \hat{\sigma}_{11} \hat{\sigma}_{22}+\ldots
$$

in such a way that the sums over $m$ and $n$ can be performed explicitly. As a consequence the cross section at fixed impact parameter, $\sigma_{H}(\beta)$, can be expressed by the operatorial form:

$$
\sigma_{H}(\beta)=\left.\left[1-\exp \left(-\delta \cdot \hat{\sigma} \cdot \delta^{\prime}\right)\right] \mathcal{Z}_{A}[J+1] \mathcal{Z}_{B}\left[J^{\prime}+1\right]\right|_{J=J^{\prime}=0}
$$


We have avoided writing explicitly the variables $u$ and $u^{\prime}$ and the functional derivative $\delta / \delta J\left(u_{i}\right)$ has been simply indicated as $\delta_{i}$.

The form of $\sigma_{H}(\beta)$ given by Eq.(2.6) is still too complicated to be worked out in its general form, since all possible multi-parton correlations are present in $\mathcal{Z}$. Therefore we further simplify the problem by taking into account two-body parton correlations only. Our explicit expression for $\mathcal{F}$ is therefore:

$$
\mathcal{F}_{A, B}[J+1]=\int D_{A, B}(u) J(u) d u+\frac{1}{2} \int C_{A, B}(u, v) J(u) J(v) d u d v
$$

where $D(u)$ is the average number of partons and $C(u, v)$ is the two-body parton correlation.

Either by using techniques of functional integration or by means of a suitable diagrammatic expansion[6] one is able to obtain in this case a closed expression for $\sigma_{H}(\beta)$ :

$$
\sigma_{H}(\beta)=1-\exp \left[-\frac{1}{2} \sum_{n} a_{n}-\frac{1}{2} \sum_{n} b_{n} / n\right]
$$

where $a_{n}$ and $b_{n}$ are functions of the impact parameter $\beta$ and are given by

$$
\begin{gathered}
a_{n}=\int D_{A}\left(u_{1}\right) \hat{\sigma}\left(u_{1}, u_{1}^{\prime}\right) C_{B}\left(u_{1}^{\prime}-\beta, u_{2}^{\prime}-\beta\right) \hat{\sigma}\left(u_{2}^{\prime}, u_{2}\right) C_{A}\left(u_{2}, u_{3}\right) \ldots \\
\ldots D_{B}\left(u_{n}^{\prime}-\beta\right) \prod d u_{i} d u_{i}^{\prime} \\
b_{n}=\int C_{A}\left(u_{n}, u_{1}\right) \hat{\sigma}\left(u_{1}, u_{1}^{\prime}\right) C_{B}\left(u_{1}^{\prime}-\beta, u_{2}^{\prime}-\beta\right) \hat{\sigma}\left(u_{2}^{\prime}, u_{2}\right) \ldots \\
\ldots C_{B}\left(u_{n-1}^{\prime}-\beta, u_{n}^{\prime}-\beta\right) \hat{\sigma}\left(u_{n}^{\prime}, u_{n}\right) \prod d u_{i} d u_{i}^{\prime} .
\end{gathered}
$$

The actual expression for $a_{n}$ (that will be referred as "open chain") holds for $n$ odd. When $n$ is odd one may also have the symmetric case, where the expression begins with $D_{B}$ and ends with $D_{A}$. When $n$ is even the initial and final distribution are either both $D_{A}$ or both $D_{B}$. In the definition of $b_{n}$, that will be referred as "closed chain" $n$ is always even, so that one of the ends is $A$ and the other is $B$. Sometimes the expression $n=2 m$ will be used. One may notice that, at a given order in the number of partonic interactions, one can obtain a term of kind $a$ from a term of kind $b$ by replacing one $C$ with a pair of $D$ 's. The operation can be done in $n$ ways. The combinatorial meaning of the $1 / n$ factor multiplying each term of kind $b$ in Eq.(2.9) is then understood. The factor $1 / 2$ in Eq.(2.9) is the consequence of the symmetry between $A$ and $B$.

The cross section is given by an integral on the impact parameter of the interaction probability, $\sigma_{H}(\beta)$, that is expressed as one minus the probability of no interaction. 
The probability of no interaction is given by the negative exponential of the sum over all possible different connected structures, namely all structures of kind $a_{n}$ and of kind $b_{n}$. With our approximations, Eq.(2.6) and Eq.(2.8), these are in fact all possible connected structures which can be built with the average numbers $D_{A, B}$, the two-body correlations $C_{A, B}$ and the interaction $\hat{\sigma}$. Expanding the exponential, the cross section can then be expressed as the sum over all possible structures, both connected and disconnected.

One will notice that, when no correlations are present, all terms of kind $b$ disappear and only the first of the terms of kind $a$, namely $D_{A} \hat{\sigma} D_{B}$ is left. In that limit the cross section is given simply by:

$$
\sigma_{H}=\int d^{2} \beta\left\{1-\exp \left[-\int D_{A}(u-\beta) D_{B}\left(u^{\prime}\right) \hat{\sigma}\left(u, u^{\prime}\right) d u d u^{\prime}\right]\right\}
$$

which corresponds to the Poissonian distribution discussed in the introduction.

\section{An explicit case: Gaussian correlation}

The bulk of the semi-hard cross section originates in the region of small $x$ values. The experimental observation on the independence of $\sigma_{e f f}$ as a function of $x$, at small $x$-values, on the other hand indicates that correlations in $x$, at small $x$, are not a strong effect in the two-body parton distribution. A sensible case to consider is therefore the one where there are no correlations in fractional momenta, nor between fractional momenta and transverse coordinates, while only transverse coordinates are correlated:

$$
D(u)=f(x) D(\mathbf{b}) \quad, \quad C\left(u, u^{\prime}\right)=f(x) f\left(x^{\prime}\right) C\left(\mathbf{b}, \mathbf{b}^{\prime}\right) .
$$

Given the localization of the partonic collisions in transverse space, the dependence of the parton-parton interaction probability on $\mathbf{b}$ and $\mathbf{b}^{\prime}$ is represented as a $\delta$-function:

$$
\hat{\sigma}\left(u, u^{\prime}\right)=\sigma_{x, x^{\prime}} \delta\left(\mathbf{b}-\mathbf{b}^{\prime}\right) .
$$

All integrations on the fractional momenta $x$ and $x^{\prime}$ are then factorized from the integrations on the transverse coordinates and result in the single scattering term cross section $\sigma_{S}$, whose expression is given in Eq.(1.5). $a_{n}$ and $b_{n}$ are therefore considerably simplified:

$$
\begin{gathered}
a_{n}=\sigma_{S}^{n} \int D_{A}\left(\mathbf{b}_{1}\right) C_{B}\left(\mathbf{b}_{1}-\beta, \mathbf{b}_{2}-\beta\right) C_{A}\left(\mathbf{b}_{2}, \mathbf{b}_{3}\right) \ldots D_{B}\left(\mathbf{b}_{n}-\beta\right) \prod d \mathbf{b}_{i} \\
b_{n}=\sigma_{S}^{n} \int C_{A}\left(\mathbf{b}_{n}, \mathbf{b}_{1}\right) C_{B}\left(\mathbf{b}_{1}-\beta, \mathbf{b}_{2}-\beta\right) \ldots C_{B}\left(\mathbf{b}_{n-1}-\beta, \mathbf{b}_{n}-\beta\right) \prod d \mathbf{b}_{i} . .
\end{gathered}
$$


To proceed further one needs to consider explicit functional forms for $C\left(\mathbf{b}, \mathbf{b}^{\prime}\right)$. A simplest extension of the Gaussian model discussed in the introduction corresponds to the following Gaussian expression of the correlation term:

$$
D(\mathbf{b})=h \exp \left[-F \mathbf{b}^{2}\right] \quad, \quad C\left(\mathbf{b}, \mathbf{b}^{\prime}\right)=k \exp \left[-\frac{1}{2} F\left(\mathbf{b}+\mathbf{b}^{\prime}\right)^{2}-\frac{1}{2} G\left(\mathbf{b}-\mathbf{b}^{\prime}\right)^{2}\right]
$$

After defining $\mathbf{b}=\mathbf{y}+\frac{1}{2} \beta, b_{n}$ is explicitly written as:

$$
b_{n}=\sigma_{S}^{n} k^{n} \int \exp \left[-\frac{1}{2} n F \beta^{2}\right] \exp \left[-(F+G) \sum \mathbf{y}_{i}^{2}\right] \exp \left[-(F-G) \sum \mathbf{y}_{i} \cdot \mathbf{y}_{i+1}\right] \prod d \mathbf{y}_{i}
$$

with the convention that the variable $\mathbf{y}_{n+1}$ coincides with $\mathbf{y}_{1}$.

$b_{n}$ can then be worked out through the substitution

$$
\mathbf{v}_{i}=\mu \mathbf{y}_{i}-\nu \mathbf{y}_{i+1} \quad ; \quad \mu-\nu=\sqrt{2 F} \quad \mu+\nu=\sqrt{2 G},
$$

which leads to

$$
b_{n}=J \sigma_{S}^{n} k^{n} \exp \left[-\frac{1}{2} n F \beta^{2}\right] \int \exp \left[-\sum \mathbf{v}_{i}^{2}\right] \prod d \mathbf{v}_{i}
$$

where the Jacobian, as discussed in Appendix B, is $J=\left(\mu^{n}-\nu^{n}\right)^{-2} . b_{n}$ is therefore finally written as:

$$
b_{n}=\sigma_{S}^{n} k^{n} \pi^{n}\left(\mu^{n}-\nu^{n}\right)^{-2} \exp \left[-\frac{1}{2} n F \beta^{2}\right] .
$$

The case of the open chain, $a_{n}$, is less symmetric and requires a slightly less straightforward treatment. Using the $\mathbf{y}$ variables, defined previously, the expression of $a_{n}$ takes the form:

$$
a_{n}=\sigma_{S}^{n} h^{2} k^{n-1} \exp \left[-\frac{1}{2} n F \beta^{2}\right] \int \exp \left[-(F+G) \mathcal{Y}^{T} \cdot \mathcal{M} \cdot \mathcal{Y}\right] \prod d \mathbf{y}_{i}
$$

where

$$
\mathcal{Y}^{T}=\left(\mathbf{y}_{1}, \mathbf{y}_{\mathbf{2}}, \ldots, \mathbf{y}_{\mathbf{n}}\right)
$$

and

$$
\begin{gathered}
\mathcal{M}=\left(\begin{array}{cccccc}
1+r & r & 0 & \ldots & 0 & 0 \\
r & 1 & r & \ldots & 0 & 0 \\
\vdots & \vdots & \vdots & \ddots & \vdots & \vdots \\
0 & 0 & 0 & \ldots & r & 1+r
\end{array}\right) \\
r=\frac{1}{2}(F-G) /(F+G) .
\end{gathered}
$$


The Gaussian integral can be evaluated (details of the calculations are reported in Appendix B) leading to the relation:

$$
a_{n}=\sigma_{S}^{n} h^{2} k^{n-1} \pi^{n} r^{n-1}\left[(2 r+1) U_{n-1}(1 / 2 r)\right]^{-1} \exp \left[-\frac{1}{2} n F \beta^{2}\right]
$$

where $U_{n}$ is the Chebyshev polynomial of second kind[7].

While the terms $a_{n}$ and $b_{n}$ are computed exactly, the sum of the series in Eq.(2.9) can be performed only in limiting cases. We discuss the case where the correlation length is much smaller with respect to the hadronic radius. The parameter $G$ characterizes the correlation while $F$ is related to the hadronic transverse size. Small correlation lengths correspond to $F<<G$. We work out therefore the leading order term in $F$ while keeping the full structure in $G$ both in $a_{n}$ and in $b_{n}$. In order to find the limiting expression of $a_{n}$ when $F<<G$, one needs to work out the limit of $U_{n}(1 / 2 r)$ for $1 / 2 r \rightarrow-1+\epsilon$. One obtains:

$$
U_{n}(-1+\epsilon) \approx(-)^{n}(n+1)
$$

By using this expression for $U_{n}$ and with similar, but simpler, manipulations one obtains the following limiting form for $a_{n}$ :

$$
a_{n}=\frac{G h^{2}}{4 n k F} Z^{n}
$$

with

$$
Z=\left(\frac{2 \pi k}{G}\right) \sigma_{S} \exp \left[-\frac{1}{2} F \beta^{2}\right]
$$

The whole series of Eq.(3.1) can be then be summed yielding as a result:

$$
S_{a}=\frac{G h^{2}}{4 k F}[-\ln (1-Z)]
$$

To work out the limiting case for $b_{n}$ we keep the exact value of the difference $(\mu-\nu)^{2}=2 F$, while setting $\mu \approx \nu \approx \sqrt{G / 2}$ everywhere else. Keeping moreover into account the condition $n=2 m$, we obtain:

$$
b_{2 m}=\frac{G}{16 F m^{2}} Z^{2 m}
$$

The resulting sum of the series in Eq.(3.1) is therefore given by

$$
S_{b}=\frac{G}{32 F} \mathcal{L}_{3}\left(Z^{2}\right)
$$

where $\mathcal{L}_{3}(x)=\sum x^{n} / n^{3}$ is the trilogarithm function[8].

The final limiting form for the cross section, when $F<<G$, is therefore: 


$$
\sigma_{H}=\int d^{2} \beta \sigma_{H}(\beta)=\int d^{2} \beta\left\{1-\exp \left[-\frac{1}{2} S_{a}-\frac{1}{2} S_{b}\right]\right\},
$$

In the limit $G \rightarrow \infty$ the correlation goes to zero. In this case one obtains $Z \rightarrow 0$, $S_{a} \rightarrow\left(\pi \sigma_{S} / 2 k F\right) \exp \left[-\frac{1}{2} F \beta^{2}\right]$ and $S_{b} \rightarrow 0$. The expression of $\sigma_{H}$ in Eq.(3.14) is then reduced to the Poissonian distribution discussed in the introduction.

\section{Two qualitatively different features of the correlation term}

The uncorrelated multiparton distributions are characterized by two independent features. One feature is that the $n$-body parton distribution is factorized in the product of $n$ times the one-body parton distribution. The second feature is that the distribution in the number of partons (namely after integrating over all other degrees of freedom) is a Poissonian. Both features are affected by the introduction of the two-body correlations and it is therefore interesting to study the two effects separately.

One may modify the number distribution, without introducing non-factorized two-body correlations in $\mathbf{b}$, by using the factorized expression

$$
C\left(u, u^{\prime}\right)=-\lambda D(u) D\left(u^{\prime}\right)
$$

The terms appearing in Eq.s $(2.10,11)$ are very much simplified in this case. After integrating over the longitudinal variables, the transverse integrations always appears in the unique form

$$
T \equiv T(\beta)=\sigma_{S} \int D_{A}(\mathbf{b}) D_{B}(\mathbf{b}-\beta) d \mathbf{b}
$$

One therefore obtains:

$$
\begin{gathered}
\frac{1}{2} \sum_{n} a_{n}=T /(1+\lambda T) \\
\frac{1}{2} \sum_{m} b_{2 m} / 2 m=\ln \left(1-\lambda^{2} T^{2}\right) .
\end{gathered}
$$

and the cross section, at a fixed value of the impact parameter, is expressed as:

$$
\sigma_{H}(\beta)=1-\left[1-\lambda^{2} T^{2}\right]^{-1 / 2} \exp [-T /(1+\lambda T)] .
$$

The parameter $\lambda$ represents a measure of the deviation from the Poissonian distribution, both for the initial states and for the elementary collisions. In Appendix $\mathrm{C}$ some further elaboration on the factorized case are presented.

Perhaps more interesting is the case where the number distribution of the incoming parton is Poissonian, because the correlation integrates to zero, while the resulting distribution in the number of parton collisions at fixed impact parameter is non-Poissonian, as a consequence of the presence of the correlation term. To study 
this case a different way of dealing with the approximation introduced in obtaining Eq.s $(3.6 ", 7 ")$ is useful.

The alternative procedure is the following: The variables $\mathbf{y}_{i}$ are substituted by the mean value $\mathbf{Y}=\sum \mathbf{y} / n$ and by the differences $\mathbf{x}_{i}=\mathbf{y}_{i+1}-\mathbf{y}_{i}$, with the constraint $\sum \mathbf{x}_{i}=0$. The relevant Jacobian is $J=1$ and so the integration volume is transformed according to: $\prod d \mathbf{y}_{i}=d \mathbf{Y} \prod d \mathbf{x}_{i} \delta\left(\sum \mathbf{x}_{i}\right)$. Then in the terms containing $F$, which simply defines the size of the hadron, one performs the substitutions $\mathbf{y}_{i} \approx \mathbf{Y} / n$ whereas the terms containing $G$ and the differences $\mathbf{x}_{i}$ are not modified. For the calculation of $a_{n}$, since only $n-1$ differences appear, the integrations in $d \mathbf{Y}$ and $d \mathbf{x}_{i}$ decouple in the Gaussian approximation and the result of Eq.s $(3.7 ", 11)$ is recovered; for the calculation of $b_{n}$ the constraint on the $\mathbf{x}_{i}$ is essential, it may be implemented in the standard exponential form $\delta\left(\sum \mathbf{x}_{i}\right)=\int(2 \pi)^{-2} \exp \left(\mathrm{i} \sum \mathbf{q} \cdot \mathbf{x}_{i}\right) d \mathbf{q}$ and carrying out all the integrations the result of Eq. (3.6") is reproduced.

This alternative calculation suggests an initial distribution which can be easily worked out and which yields to a pure Poissonian distribution in the number of partons in the initial states. One may in fact consider the following expressions:

$$
D(\mathbf{b})=h \exp \left[-F \mathbf{b}^{2}\right], C\left(\mathbf{b}, \mathbf{b}^{\prime}\right)=-k^{\prime} \Delta_{2} \exp \left[-\frac{1}{2} F\left(\mathbf{b}+\mathbf{b}^{\prime}\right)^{2}-\frac{1}{2} G\left(\mathbf{b}-\mathbf{b}^{\prime}\right)^{2}\right]
$$

where $\Delta_{2}$ indicates the two-dimensional Laplace operator acting on the transverse difference $\mathbf{b}-\mathbf{b}^{\prime}$ or $\mathbf{y}-\mathbf{y}^{\prime}$. By integrating $C$ over the transverse variables one obtains zero. The number distribution depends therefore only on $D$ and it is Poissonian by construction. The parameter $k^{\prime}$ is obviously related to the parameter $k$ in the previous paragraph. $k$ was the strength of the correlation at $\mathbf{b}=\mathbf{b}^{\prime}=0$. To keep the same normalization we make the position $k^{\prime}=k / G$.

The contribution of all open chains $a_{n}$ with $n>1$ is therefore zero. As previously observed in this case the differences have no constraint. Every independent integration over a transverse difference corresponds therefore to an integration of a single correlation term, which gives zero. Small nonzero effects have been possibly lost in the approximate substitution $\mathbf{y}_{i} \approx \mathbf{Y} / n$ in the terms containing $F$ in Eq.'s(3.6', $\left.7^{\prime}\right)$.

When one computes the contribution of the closed chain $b_{n}$, the constraint is effective. The calculation is again performed by means of the exponential representation of the $\delta$-function and the resulting expression can be brought to the following form

$$
\tilde{b}_{2 m}=\frac{G}{8 F m^{2}}(Z / m)^{2 m}(2 m) !
$$

The final result is obtained by summing all the terms above, with the weight $(1 / 2 m)$. The cross section (at $\beta$ fixed) is therefore

$$
\sigma_{H}(\beta)=1-\exp \left[-T-\tilde{S}_{b}\right] \quad, \quad \tilde{S}_{b}=\sum_{m=1}^{\infty} \frac{\tilde{b}_{2 m}}{2 m}
$$


While a closed analytic form for the sum $\tilde{S}_{b}$ is not easily written in the general case, in the limiting case where the number of partonic collisions is large one may use the Stirling approximation for the factorial: $m^{-m} \approx \sqrt{2 \pi m} \mathrm{e}^{-m} / m$ !. With the help of the decomposition $(2 m) !=2^{2 m} m !\left(\frac{1}{2}\right)_{m}$ the sum can be expressed as

$$
\tilde{S}_{b}=\frac{\pi G}{8 F} \psi(w)
$$

where

$$
w=(2 Z / \mathrm{e})^{2} \quad, \quad \psi(w)=\sum_{1}^{\infty} \frac{\left(\frac{1}{2}\right)_{m}}{m^{2} m !} w^{m}
$$

$\psi(w)$ can be evaluated by computing, as intermediate step,

$$
\left(w \partial_{w}\right)\left(w \partial_{w}\right) \psi(w)=\sum_{1}^{\infty} \frac{\left(\frac{1}{2}\right)_{m}}{m !} w^{m},
$$

and by performing the integrations afterwards. The resulting expression is:

$$
\psi(w)=2 \mathcal{L}_{2}\left(\frac{1}{2}[1-\sqrt{1-w}]\right)-\ln ^{2}\left(\frac{1}{2}[1+\sqrt{1+w}]\right) .
$$

where $\mathcal{L}_{2}(x)=\sum x^{n} / n^{2}$ is the dilogarithm function.

\section{Concluding discussion}

The small value of $\sigma_{e f f}$, the dimensional parameter characterizing double parton scatterings, which has been measured recently by CDF, is an indication that two-body parton correlations, in the many-body parton distribution of the proton, are likely to be sizeable. In the case of an uncorrelated many-body parton distribution, the value of $\sigma_{e f f}$ puts a constraint on the range of possible values of $\sigma_{H}$, the semi-hard contribution to the hadronic inelastic cross section. The actual measured value of $\sigma_{\text {eff }}$ would give rise to values of $\sigma_{H}$ of the order of $\sigma_{\text {inel }} / 2$ also at very large c.m. energies, where one would rather expect $\sigma_{H} \simeq \sigma_{\text {inel }}$. The experimental evidence is also that, in the $x$ region accessible experimentally namely at small $x$ values, the correlation in fractional momenta is not a large effect.

In the present paper we have worked out the semi-hard cross section $\sigma_{H}$ in the case where initial state partons are correlated in the transverse parton coordinates. In fact $\sigma_{H}$ can be worked out rather explicitly when only two-body parton correlations are included in the many-body parton distributions and when each parton can have at most one semi-hard interaction. There are two qualitatively different features in the two-body parton correlation, and both change the relation between $\sigma_{H}$ and $\sigma_{e f f}$ with respect to the uncorrelated case: 
1- The distribution in the number of partons is not any more a Poissonian, although the dependence on the kinematical variables of the different partons is factorized.

2- The overall distribution in the number of partons, namely after integrating on the partonic kinematical variables, is a Poissonian but the dependence on the partonic kinematical variables is not factorized, in which case the two-body parton correlation integrates to zero.

The general case is obviously a combination of the two possibilities. We point out however that both cases separately can give rise to a small value of $\sigma_{\text {eff }}$ while keeping the value of $\sigma_{H}$ close to $\sigma_{\text {inel }}$.

In the first case $\sigma_{H}$ is obtained by integrating the expression in Eq.(4.3). In the second by integrating the expression in Eq.(4.6). The critical value of the impact parameter $\beta_{c}$, which gives the size to the cross section $\sigma_{H}$, is the value which makes small the argument of the exponential in the expression of $\sigma_{H}(\beta)$. The detailed dependence of the argument of the exponential at $\beta<\beta_{c}$ is not of great importance for the determination of $\sigma_{H}$. It only determines the degree of blackness, with respect to semi-hard interactions, of the overlapping hadronic matter at the given value of the impact parameter. It is therefore enough to determine the argument of the exponential at the edge of the interaction region. In case 1 the behaviour of the argument of the exponential when going at the edge of the interaction region corresponds to the limiting behaviour of the expression below at small $T$ values:

$$
\frac{T}{1+\lambda T}+\frac{1}{2} \ln \left(1-\lambda^{2} T^{2}\right) \rightarrow T=\sigma_{S} \int D_{A}(\mathbf{b}) D_{B}(\mathbf{b}-\beta) d^{2} b
$$

while in case 2 it corresponds to neglect $\tilde{S}_{b}$ as compared with $T$ in Eq.(4.6):

$$
T+\tilde{S}_{b} \rightarrow T=\sigma_{S} \int D_{A}(\mathbf{b}) D_{B}(\mathbf{b}-\beta) d^{2} b .
$$

In both cases the critical value $\beta_{c}$ has the same value which one finds for the uncorrelated distribution and therefore $\sigma_{H}$, as in the uncorrelated case, is roughly equal to $2 \pi / F$, where $F$ is the parameter giving the extension of the one-body parton distribution in the transverse plane.

When the double scattering cross section is worked out by expanding the expression of $\sigma_{H}$, one finds for the effective cross section the relation

$$
\sigma_{e f f}=\frac{2 \pi}{F \cdot(1+\lambda)^{2}}
$$

in case 1 , and

$$
\frac{1}{\sigma_{e f f}}=\frac{F}{2 \pi}+\int C\left(\mathbf{b}, \mathbf{b}^{\prime}\right) C\left(\mathbf{b}-\beta, \mathbf{b}^{\prime}-\beta\right) d^{2} b d^{2} b^{\prime} d^{2} \beta
$$

in case 2. 
A qualitative feature is that in both cases one obtains a value of $\sigma_{\text {eff }}$ which may be sizably smaller with respect to $2 \pi / F \simeq \sigma_{H}$. While, on the other hand, nothing prevents the value of $\sigma_{H}$ to be close to the value of $\sigma_{\text {inel }}$. The smaller value of $\sigma_{\text {eff }}$, with respect to the expectation of the uncorrelated case, is rather generally associated with the increased dispersion of the distribution in the number of partonic collisions: In the case of no correlations the distribution is strictly Poissonian when the impact parameter is fixed. When correlations are introduced the distribution in the number of parton collisions, at fixed $\beta$, is not Poissonian any more and the natural consequence is that the dispersion in the number of collisions is increased.

The indication from the measure of the rate of double parton scatterings is therefore that two-body parton correlations are likely to be important while, unfortunately, one cannot say much about dynamical quantities, like the the correlation length. Useful observables to be measured, in order to get some more insight into the problem, would be the semi-hard cross section $\sigma_{H}$ and the triple parton scattering cross section. Our present analysis shows that $\sigma_{H}$ can be reliably discussed in perturbation theory. The measure of $\sigma_{H}$, in association with $\sigma_{e f f}$, would help considerably in clarifying the size of the effect induced by the presence of the two-body parton correlations: All the considerations of the present paper are based on the prejudice that $\sigma_{H}$ should have a value rather close to the value of $\sigma_{\text {inel }}$.

The measure of triple parton scattering would allow one to test the possibility discussed in case 1, since in that case the rate of triple scatterings would be strictly fixed by the measured rate of double scatterings. In the other cases presently discussed the knowledge of the rate of triple parton collisions would allow one to obtain the actual values of the parameters of the correlation term.

A lot of effort has been put on the study of the proton structure as a function of the momentum fraction $x$. The distribution of partons however depends on three degrees of freedom, the momentum fraction $x$ and the transverse parton coordinate $\mathbf{b}$. The measure of the rate of triple and of higher order partonic collisions is the essential tool to learn on the parton structure of the proton in transverse plane.

\section{Acknowledgements}

We thank M. Strikman for useful comments and discussions.

This work was partially supported by the Italian Ministry of University and of Scientific and Technological Research by means of the Fondi per la Ricerca scientifica - Università di Trieste. 


\section{Appendix A}

In this appendix some short observations about the effects of the rescattering processes will be displayed. The general form of the hadron-hadron scattering cross section Eq. (2.5) has been reduced to a more manageable form by throwing away all the rescattering process by means of the position:

$$
\left\{1-\prod_{i, j}^{n, m}\left[1-\hat{\sigma}_{i j}\right]\right\} \Rightarrow \sum_{i j} \hat{\sigma}_{i j}-\frac{1}{2 !} \sum_{i j} \sum_{k \neq i, l \neq j} \hat{\sigma}_{i j} \hat{\sigma}_{k l}+\ldots
$$

It is possible to deal with some rescattering process without too much effort if we look at processes where only one of the two colliding partons has already suffered some collision. In this case instead of Eq. (A.1) we write

$$
\left\{1-\prod_{i, j}^{n, m}\left[1-\hat{\sigma}_{i j}\right]\right\} \Rightarrow \sum_{i j} \hat{\sigma}_{i j}-\frac{1}{2 !} \sum_{i j}\left\{\sum_{k \neq i, l} \hat{\sigma}_{i j} \hat{\sigma}_{k l}+\sum_{k, l \neq j} \hat{\sigma}_{i j} \hat{\sigma}_{k l}-\sum_{k \neq i, l \neq j} \hat{\sigma}_{i j} \hat{\sigma}_{k l}\right\}+\ldots
$$

In this expression the first term shows that the partons belonging to the first hadron suffer only one collision, the partons belonging to the second hadron undergo any number of collisions, the second term describes the symmetrical situation, the third term eliminates the double counting of the single-collision processes. ${ }^{*}$.

The operatorial form of the cross section at fixed impact parameter $\sigma_{H}(\beta)$ acquires now the more complicated shape:

$$
\begin{gathered}
\sigma_{H}(\beta)=\left[1-\exp \left(\delta \cdot\left[\mathrm{e}^{\hat{\sigma} \cdot \delta^{\prime}}-1\right]\right)-\exp \left(\delta^{\prime} \cdot\left[\mathrm{e}^{\hat{\sigma} \cdot \delta}-1\right]\right)+\exp \left(-\delta \cdot \hat{\sigma} \cdot \delta^{\prime}\right)\right] \times \\
\left.\mathcal{Z}_{A}[J+1] \mathcal{Z}_{B}\left[J^{\prime}+1\right]\right|_{J=J^{\prime}=0}
\end{gathered}
$$

This expression seems of uneasy interpretation but is gives some information when reduced to simpler particular cases The simplest, but non trivial result is produced when the incoming parton distribution is purely Poissonian and only the double scattering is actually considered. In these situations it is easily seen that instead of Eq. (2.10) one obtains the form:

$$
\begin{aligned}
\sigma_{H}= & \int d \beta\left\{1-\exp \left[-\int D_{A}(u) \hat{\sigma}\left(u, u^{\prime}\right) D_{B}\left(u^{\prime}\right) d u d u^{\prime}\right] \times\right. \\
& \left(\exp \left[\frac{1}{2} \int D_{A}(u) \hat{\sigma}\left(u, u^{\prime}\right) \hat{\sigma}\left(u, u^{\prime \prime}\right) D_{B}\left(u^{\prime}\right) D_{B}\left(u^{\prime \prime}\right) d u d u^{\prime} d u^{\prime \prime}\right]+\right. \\
& \left.\left.\exp \left[\frac{1}{2} \int D_{A}(u) D_{A}\left(u^{\prime \prime}\right) \hat{\sigma}\left(u, u^{\prime}\right) \hat{\sigma}\left(u^{\prime \prime}, u^{\prime}\right) D_{B}\left(u^{\prime}\right) d u d u^{\prime} d u^{\prime \prime}\right]-1\right)\right\}
\end{aligned}
$$

\footnotetext{
* This possibility was already considered in ref [6], for an unsymmetrical situation
} 
This form is not unexpected, it could have been written by hand, Eq.(A.3) shows a more systematic way of deriving it and possible further corrections. The final distribution of the collision is not Poissonian and this deviation is purely due to the hard dynamics. It can be observed that precisely this origin in the hard collision may also offer a way to distinguish these effects from the effects of correlations in the incoming two-body distributions, in fact in the case here sketched there should be an unbalance among the kinematical variables of the pair of jets originating from the scattered partons.

\section{Appendix B}

The Jacobian appearing in Eq.(3.6), which arises from the transformation from the variables $\mathbf{y}$ to the variables $\mathbf{v}$, is expressed as $J=[\operatorname{det} \mathcal{J}]^{-2}$. The actual form of $\mathcal{J}$ is:

$$
\mathcal{J}=\left(\begin{array}{cccccc}
\mu & -\nu & 0 & \ldots & 0 & 0 \\
0 & \mu & -\nu & \ldots & 0 & 0 \\
\vdots & \vdots & \vdots & \ddots & \vdots & \vdots \\
-\nu & 0 & 0 & \ldots & 0 & \mu
\end{array}\right)
$$

and the result $\operatorname{det} \mathcal{J}=\mu^{n}-\nu^{n}$ is easily obtained by expansion according to the last row. The exponent -2 appears because the matrix describes the inverse transformation and there are two transverse dimensions.

In order to calculate the determinant of the matrix $\mathcal{M}$ in Eq.(3.7) an auxiliary matrix $\mathcal{A}$ is introduced so that we have

$$
\mathcal{M}=\left(\begin{array}{cccccc}
1+r & r & 0 & \ldots & 0 & 0 \\
r & 1 & r & \ldots & 0 & 0 \\
\vdots & \vdots & \vdots & \ddots & \vdots & \vdots \\
0 & 0 & 0 & \ldots & r & 1+r
\end{array}\right) \quad, \quad \mathcal{A}=\left(\begin{array}{cccccc}
1 & r & 0 & \ldots & 0 & 0 \\
r & 1 & r & \ldots & 0 & 0 \\
\vdots & \vdots & \vdots & \ddots & \vdots & \vdots \\
0 & 0 & 0 & \ldots & r & 1
\end{array}\right)
$$

By means of the standard rules for the computation of the determinants two recurrence relations are obtained:

$$
\begin{gathered}
\operatorname{det} \mathcal{M}_{n}=\operatorname{det} \mathcal{A}_{n}+2 r \operatorname{det} \mathcal{A}_{n-1}+r^{2} \operatorname{det} \mathcal{A}_{n_{2}} \\
\operatorname{det} \mathcal{A}_{n}=\operatorname{det} \mathcal{A}_{n-1}-r^{2} \operatorname{det} \mathcal{A}_{n_{2}}
\end{gathered}
$$

which imply the simpler relation

$$
\operatorname{det} \mathcal{M}_{n}=(2 r+1) \operatorname{det} \mathcal{A}_{n-1}
$$

The recurrence relations for the determinants of $\mathcal{A}_{n}$ are very similar to the recurrence relation for the Chebyshev polynomials $U_{n}[7]$ and in this way it is possible to get the final expression for them

$$
\operatorname{det} \mathcal{A}_{n}=r^{n} U_{n}(1 / 2 r)
$$


For completeness we give also the explicit expression of the $U$-polynomials:

$$
U_{n}(\cos \theta)=\frac{\sin (n+1) \theta}{\sin \theta} .
$$

\section{Appendix C}

In this appendix we shall briefly discuss some non-Poissonian one-body densities, the essential point were already shown in ref.[6], but it may be useful to state again them in order to have a comparison of the previous treatment. The starting point of Eq.(2.7) is specialized to the case of pure one-body densities in the form

$$
\sigma_{H}=\left.\left(1-\exp T \partial \partial^{\prime}\right) X_{A}(\mathcal{Y}) X_{B}\left(\mathcal{Y}^{\prime}\right)\right|_{\mathcal{Y}=\mathcal{Y}_{o}, \mathcal{Y}^{\prime}=\mathcal{Y}^{\prime}{ }_{o}} .
$$

The Poissonian distribution is given by $X=\exp \left[\mathcal{Y}-\mathcal{Y}_{o}\right]$, the situation described in Eq.(4.1) corresponds to

$$
X=\exp \left[\mathcal{Y}-\mathcal{Y}_{o}\right]+\frac{1}{2} \lambda\left[\mathcal{Y}-\mathcal{Y}_{o}\right]^{2},
$$

Then by applying Eq.(C.1) to Eq.(C.2), one gets as intermediate step

$$
\exp \left[-T \partial^{\prime}+\frac{1}{2} \lambda\left(T \partial^{\prime}\right)^{2}\right] \exp \left[\mathcal{Y}^{\prime}-\mathcal{Y}_{o}^{\prime}\right]+\left.\frac{1}{2} \lambda\left[\mathcal{Y}^{\prime}-\mathcal{Y}_{o}^{\prime}\right]^{2}\right|_{\mathcal{Y}^{\prime}}=\mathcal{Y}^{\prime}{ }_{o},
$$

By the use of the general formula:

$$
\exp \left[-p \partial_{x}^{2}\right] \exp \left[q x^{2}\right]=\frac{1}{\sqrt{1-4 p q}} \exp \left[\frac{q x^{2}}{1+4 p q}\right]
$$

it is seen that the expression appearing in Eq.(C.3) reduces to the form already given in Eq.(4.3).

One could also choose the form

$$
X=\left[1-\left(\mathcal{Y}-\mathcal{Y}_{o}\right)\right]^{-\alpha}
$$

which corresponds to a negative binomial distribution for the initial partons and obtain in this way the result

$$
\sigma_{H}(\beta)=1-T^{-\alpha} U[\alpha ; 1 ; 1 / T],
$$

by representing the incoming distributions as Laplace transforms as shown in ref.[6], note that a slight simplification has been introduced in the distribution, with respect to that reference, and this reflects into a slight simplification of the result, which keep however its main properties, in particular $U$ is the irregular confluent hypergeometric function. 


\section{References}

1. F. Abe et al. (CDF Collaboration), submitted to Phys. Rev. D April 14, 1997; FERMILAB-PUB-97/094-E.

2. C. Goebel, F. Halzen and D.M. Scott, Phys. Rev. D22, 2789 (1980); N. Paver and D. Treleani, Nuovo Cimento A70, 215 (1982); B. Humpert, Phys. Lett. B131, 461 (1983); B. Humpert and R. Odorico, ibid 154B, 211 (1985); T. Sjostrand and M. Van Zijl, Phys. Rev. D36, 2019 (1987).

3. T. Akesson et al. (AFS Collaboration), Z. Phys. C34 163 (1987); J. Alitti et al. (UA2 Collaboration), Phys. Lett B268 145 (1991); F. Abe et al. (CDF Collaboration), Phys. Rev. D47 4857 (1993).

4. Ll. Ametller and D. Treleani, Int. J. Mod. Phys. A3, 521 (1988).

5. V. Abramovskii, V.N. Gribov and O.V. Kancheli, Yad. Fiz. 18, 595 (1973) [Sov. J. Nucl. Phys. 18, 308 (1974) ]; I.G. Halliday and C.T. Sacharajda, Phys. Rev. D8, 3598 (1973); J. Koplik and A.H. Mueller, Phys. Lett. 58B, 166 (1975); L.D. Mc Lerran and J.H. Weiss, Nucl. Phys. B100, 329 (1975); L. Bertocchi and D. Treleani, J. Phys. G3 147 (1977); V.M. Braun and Yu.M. Shabelski, Int. J. Mod. Phys. A3, 2417 (1988); G. Calucci and D. Treleani Phys. Rev. D49, 138 (1994); D50, 4703 (1994); J. Bartels and M. Wüsthoff, Z. Phys. C66, 157 (1995); D. Treleani, Int. J. Mod. Phys. A11 613 (1996).

6. G. Calucci and D. Treleani, Nucl. Phys. B (Proc. Suppl.) 18C, 187 (1990) and Int. J. Mod. Phys. A6, 4375 (1991).

7. M. Abramowitz and I. A. Stegun, Handbook of Mathematical Functions, Dover Publications, Inc., New York.

8. L. Lewin, Dilogarithms and Associated Functions, Macdonald, London (1985). 